\title{
A Heterogeneous Environment for Computational Prototyping and Simulation Based Design of MEMS Devices
}

\author{
N.M. Wilson, Z. K. Hsiau, R. W. Dutton, and P. M. Pinsky \\ Departments of Electrical and Mechanical Engineering \\ TCAD Group and Division of Mechanics and Computation \\ CISX 332, Stanford University,Stanford, CA 94305-4075
}

\begin{abstract}
This paper gives an overview of MEMS simulation and the computational requirements in the field from the prospective of creating realistic geometry based on process simulation. We motivate the needs for such geometry, and discuss in detail one approach to create pseudo 3-D geometry from 2-D process simulation.
\end{abstract}

\section{Introduction}

Micro-Electro-Mechanical Systems (MEMS) offer dramatic new functional capabilities. Analysis of MEMS is a challenge; the structures are geometrically complicated, electromechanically coupled, and inherently three-dimensional. 'The multi-domain physics for MEMS includes: electrical, mechanical, and fuidic interactions. Effects of each domain need to be accurately simulated to correctly predict device performance. In addition, IC-based fabrication techniques require the modeling of complex material belavior and processing such as etching, deposition, and thermal processes such as diffusion (and oxidation).

\section{Computational Approaches}

Two quite different modeling methodologies are being pursued among the MEMS community. The "homogeneous" approach is to develop tightly integrated component simulation tools and design methodology. Alternatively, a "heterogeneous" approach is one that wraps distinctly different end-user software packages to create a "meta" tool. The MEMCAD system is one well known example that uses commercial and other academic tools (ABAQUS, IDEAS, and Fastcap) [1].

\section{Exploiting Complex Geometry}

Currently available heterogeneous implementations have limitations especially in the area of creating and utilizing complex geometry. A geometry server is demonstrated, based on a solid modeler [1], that permits the efficient integration of process simulation 
results into a portable geometric model. The incorporation of $h p$-adaptivity is critical for analysis of many components. The present work demonstrates simulations where complex geometry is used in an environment that provides sophisticated anisotropic $h p$-adaptivity [1].

\section{Previous utilization of Solid Modeling in TCAD}

There have been efforts in the past to use solid modeling for VLSI applications [2]. These methods rely on the user providing simulation results, sweeping options, and process flow information. The major difference between the approach described in this paper and the other efforts is that the process simulation is run automatically for the user. In addition to being run automatically, the process simulation input is actually generated from the geometry as the model is being built. The goal is to automate the process.

\section{Geometry Modeling Support}

Robust 3-D process simulators do not currently exist to model deposition and etching. A new pscudo 3-D physical process simulation approach is demonstrated which is fully integrated with traditional geometric capabilities. The technique used to create $3-\mathrm{D}$ physical geometry consists of five major steps:

1) Create an initial geometry.

2) Cut the geometry into "slices" in the $x$ and $y$ planes.

3) Perform 2-D physical simulation on the "slices" using SPEEDIE [1].

4) For the results in each direction, a 2 nd degree nonuniform rational b-spline (NURBS) is created.

5) A boolean addition (union) of the two NURBS is then performed to generate the final 3-D profile.

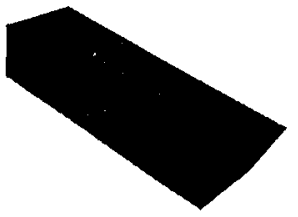

(a) initial geometry.

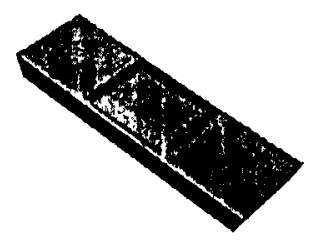

(d) nurb in the $x$ direction.

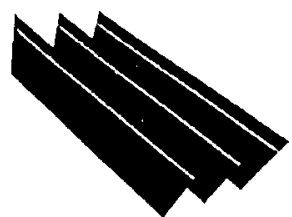

(b) cuts in the $x$ direction.

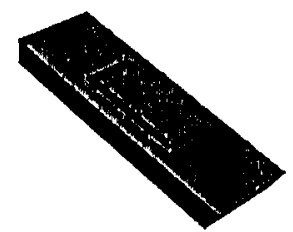

(c) nurb in the $y$ direction.

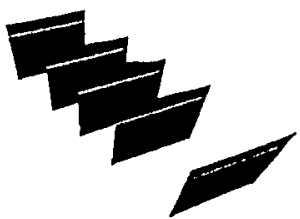

(c) cuts in the y-direction.

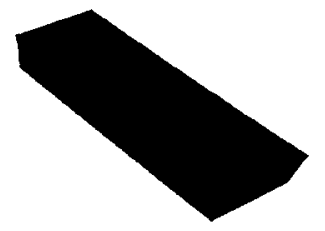

(f) unioned gcometry showing deposition. 


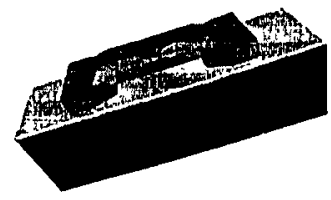

(g) view of RF switch.

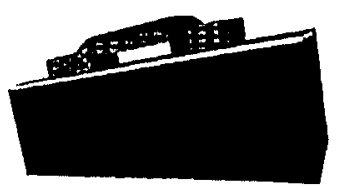

(h) view of RF switch.

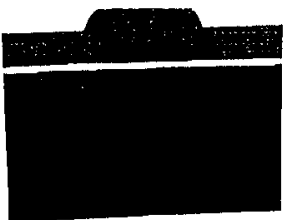

(i) selected cross section showing rounded corners due to SPEEDIE simulation.

Figure 1: Steps to create pseudo 3-D geometry.

The resulting profile is incorporated into the solid model, and can be manipulated in subsequent steps by geometric operations and additional physical depositions. The RF switch shown (Fig. 1) was created using this technique. The entire device was created by the user defining only the mask specification (.CIF file) and a simple process flow. One physical deposition step was performed in conjunction with multiple geometric etches to achieve the final shape. Notice the rounded corners on the top of the switch due to the physical process simulation, while the sharp edges are the result of geometric etching.

The main advantage of using the union of the two NURBS is that it yields a smoothing of the intersection of $x$ plane and $y$ plane simulations. This topological smoothing of the 3-D effects is the best that can be achieved without full 3-D simulation. Major computational cost savings is thereby achieved. The drawback with using NURBS is that the union of the NURBS is a complex intersection problem, which the solid modeler being used cannot handle in a robust fashion. Current work includes evaluating another solid modeler and using a triangulized surface representation of the solids for unioning.

\section{Challenges in Geometry - Corners and Number of Cuts}

While corner effects for VLSI may be critical, in some MEMS structures of interest corner effects play a less dominate role. A practical example would be the new stepup anchor discussed in [3]. Fig. 2 indicates the current performance of the method at a corner. As you would expect, the method predicts reasonable cover on the step, but inaccurately predicts no coverage in the region labeled $\beta$. One possibility being explored to address this problem is using an extra "angled" slice plane (show as a dotted line) which would give a more reasonable result in this region.

The other significant area for improvement is to automatically detect the number of cut planes being used in the $x$ and $y$ directions. Currently, the user specifies the number of cut planes in the $x$ and $y$ direction and the program uses uniformly spaced cuts. This causes two potential difficulties in that the certain features could be missed if insufficient refinement is used and that unnecessary simulation is also performed due to the non-optimal spacing of the cut planes. The image shown of the RF switch was created using $40 \times 40$ cuts, while with non-uniform spacing this number could be reduced to $6 \times 6$ for the same level of accuracy. 


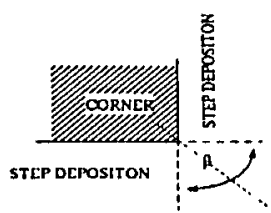

(a) schematic showing (top) view.

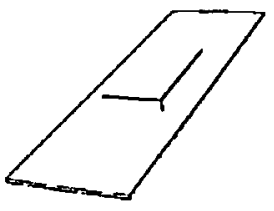

(b) before deposition.

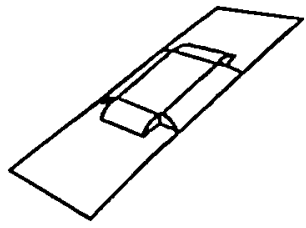

(c) after deposition.

Figure 2: Corner effects.

\section{Extensions in Simulation}

Important multi-physics interactions and numerical requirements that need to be addressed in dynamic response simulation of MEMS systems are a key focus of prototype simulation development efforts. Current progress includes the demonstration of a versatile framework to test MEMS requirements for solving coupled systems of equations using ProPHLEX [1] as a prototype vehicle. The solution of coupled elastostatic-electrostatic equations is demonstrated based on a relaxation scheme; ongoing work is targeted to exploit $h p$-adaptivity using gradient based error predictors. Fig. 3 shows the Von Mises stress for the electrostatically actuated RF switch. Stress concentrations occur at the inner corners due to the sharp edges. Note that a similar result would have been seen at the top edges if geometric deposition had been performed. Physically based simulation, however, indicates rounded corners will appear and thus no stress concentration will be observed at the top corners. This is in betler agreement with experimental results.

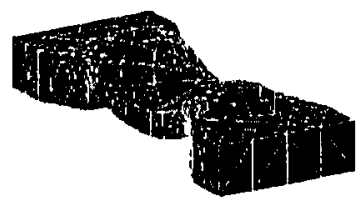

Figure 3: Von-Mises stress distribution of the electrostatically actuated RF switch.

Acknowledgments The authors would like to thank David Parker for his contributions and support of the solid modeling efforts. This work is supported under Air Force/DARPA No. F30602-96-2-0308.

\section{References}

[1] SPEEDIE, http://speedic.stanford.edu. MEMCAD, http://www.memcad.com. Shapes, http://www.xox.com. ProPHLEX, http://www.comco.com.

[2] K. Wang, H.S. Park, Z. Yu, R.W. Dutton, "3D Solid Modeling of IC Structures Using Simulated Surface Topography," SISPAD Conference Proceedings, Tokyo, 1996, pp. 131-132.

[3] J. Jung-Yeul Gill, L.V. Ngo, P.R. Nelson, and C.J. Kim, "Elimination of Extra Spring Effect at the Step-Up Anchor of Surface-Micromachined Structure", J. Microclectromechanical Sys., vol. 7, no. 1, pp. 114-123. 